EPJ Web of Conferences 41, 04019 (2013)

DOI: $10.1051 /$ epjconf/20134104019

(C) Owned by the authors, published by EDP Sciences, 2013

\title{
Manipulation of Squeezed Two-Phonon Bound States using Femtosecond Laser Pulses
}

\author{
Jianbo $\mathrm{Hu}^{1,2}$, Oleg V. Misochko ${ }^{3}$, and Kazutaka G. Nakamura ${ }^{1,2}$ \\ ${ }^{1}$ Materials and Structures Laboratory, Tokyo Institute of Technology, R3-10, 4259 Nagatsuta, \\ Yokohama 226-8503, Japan \\ ${ }^{2}$ CREST, Japan Science and Technology Agency, Kawaguchi 332-0012, Japan \\ ${ }^{3}$ Institute of Solid State Physics, Russian Academy of Sciences, 142432 Chernogolovka, Moscow \\ region, Russia
}

\begin{abstract}
Two-phonon bound states have been excited exclusively in $\mathrm{ZnTe}(110)$ via impulsive stimulated second-order Raman scattering, essentially being squeezed states due to phase coherent excitation of two identical components anticorrelated in the wave vector. By using coherent control technique with a pair of femtosecond laser pulses, the manipulation of squeezed states has been demonstrated in which both the amplitude and lifetime of coherent oscillations of squeezed states are modulated, indicating the feasibility to control the quantum noise and the quantum nature of phonon squeezed states, respectively.
\end{abstract}

\section{Introduction}

Both theories and experiments have demonstrated that phonon squeezed states can be generated via the impulsive stimulated second-order Raman scattering [1-4]. In such a state, the uncertainty of one variable is reduced to the level lower than the phonon vacuum state, overcoming the limitation of the uncertainty principle. Due to coherenceof the phonon squeezed states created by ultrashort laser pulses, in principle, it is possible to actively manipulate this quantum state using pulse shaping or pulse-train techniques.

Coherent LO phonons at $6.15 \mathrm{THz}$ and a second-order Raman mode at $3.5 \mathrm{THz}$ have been excited in $\mathrm{ZnTe}(100)$ [5]. The pump-polarization dependence of the LO mode suggests that it is possible to excite the second-order phonon mode alone by selecting $\mathrm{ZnTe}(110)$ as the sample.

In this work, we employ a pair of pump pulses to manipulate squeezed two-phonon bound states (TPBS) in $\mathrm{ZnTe}(110)$, aiming to further reduce the quantum fluctuations of the atomic displacements.

\section{Experimental}

All experiments were made using a pump-probe transmission-type setup at room temperature. The sample was a $10 \times 10 \times 1 \mathrm{~mm}^{3}$ high-quality ZnTe(110) single crystal. A mode-locked Ti-sapphire laser provided pulses centered at $800 \mathrm{~nm}$ with their FWHM duration of $40 \mathrm{fs}$. The pump and probe beams have orthogonal polarizations, both focused to a $50-\mu \mathrm{m}$-diameter spot. For the two-pump excitation a

This is an Open Access article distributed under the terms of the Creative Commons Attribution License 2.0, which permits unrestricted use, distribution, and reproduction in any medium, provided the original work is properly cited. 
stronger pump pulse was fed into a modified Mach-Zehnder interferometer and divided into two collinear pulses (of 23 and $30 \mathrm{~mW}$ average power) with a variable interpulse separation. We measured the real-time transmission change $\left(\Delta T / T_{0} \equiv\left(T-T_{0}\right) / T_{0}\right)$ of the probe pulse after the ultrafast excitation of $\mathrm{ZnTe}(110)$ by using a homodyne-like difference-detection scheme, where $\mathrm{T}$ and $\mathrm{T}_{0}$ are the transmission with and without pump excitation, respectively.

\section{Results and discussion}

The single pump excitation measurements show that we excited exclusively two-acoustic phonon bound states in ZnTe(110), see Fig.1, which have a slightly higher frequency than the corresponding overtone $2 \mathrm{TA}(\mathrm{X})[6]$. The amplitude of coherent oscillation is linearly dependent on the pump power and independent on the pump polarization, indicating the excitation mechanism of impulsive stimulated second-order Raman scattering and the entity of a full symmetry, respectively. The physical essence of the TPBS excitation is the divergence in the phonon density of states at the zone boundary due to Van Hove singularity that allows effectively pump energy into acoustic band. It is residual phonon-phonon interaction that causes the bound state to split off from above the band of free (unbound) two-phonon states [7].
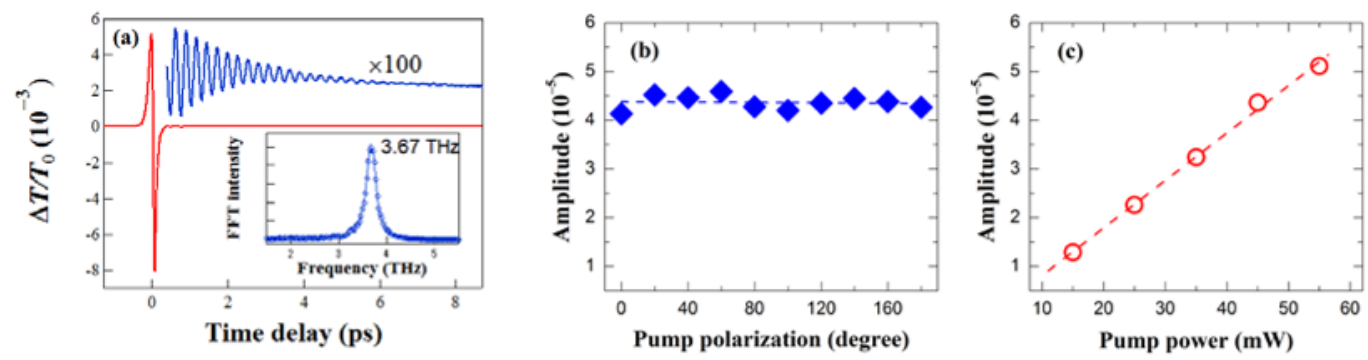

Fig. 1. Evidences of the assignment of two-phonon bound states. (a) the time-domain signal with singlefrequency coherent oscillation; (b) the independence of the oscillatory amplitude on the pump polarization; (c) the linear dependence of the oscillatory amplitude on the pump power.

TPBS are composed of two acoustic phonons characterized by equal frequencies and opposite wave vectors. Due to phase coherent and simultaneous excitation, two component phonons of TPBS interfere destructively, resulting in the zero population of the first-order Raman mode. Therefore, the average atomic displacement is reduced to zero $\left\langle Q_{q}(t)>=0\right.$, and the transmission change is directly related to the quantum fluctuation of the atomic displacements [3],

$$
\Delta T \equiv T-T_{0}=\sum_{q} \frac{\partial^{2} T}{\partial Q_{q} \partial Q_{-q}}\left\langle Q_{q} Q_{-q}\right\rangle=\sum_{q} \frac{\partial^{2} T}{\partial Q_{q} \partial Q_{-q}}\left\langle\Delta u^{2}( \pm q, t)\right\rangle
$$

where $q$ is the wave vector, $Q$ the normal mode operator, $u$ the atomic displacement, and the average is over the phonon states, the fluctuation is modulated at the twice frequency of the constituent mode, squeezing at some specific moments. TPBS, therefore, are referred to squeezed states. Intuitively, by controlling the probed transmission change, one may realize the manipulation of the phonon squeezed state.

Due to coherence created by ultrashort laser pulse, coherent oscillations of TPBS can be readily controlled. To this end, we used two collinear pump pulses with a variable interpulse separation to modify TPBS. The first pump pulse starts a coherent motion with all atoms moving in a synchronous manner, then the second pump pulse excites a phase-delayed replica of the first and finally the two interfere. Therefore, by varying the phase difference (that is interpulse separation), it is possible to bring the superposition either to a destructive or a constructive interference and thus to control the final state attained after the double pulse excitation. The change in the two-phonon amplitude and lifetime as the interpulse separation was varied is shown in Fig. 2. As seen from this figure, the 
resulting coherent amplitude in ZnTe varies almost harmonically in a way that the TPBS can be either significantly enhanced or almost suppressed, which happens at integer and half-integer oscillatory periods, respectively. It means that we have realized the manipulation of phonon squeezed states. By the coherent control, we can steer the lattice into two opposite states: one corresponds to the situation $(\Delta t=(\mathrm{n}+1 / 2) T \mathrm{c}, \mathrm{n}$ is the integer) when all atoms are almost at equilibrium position (the fluctuation of atomic displacements is small), while their momentum distribution is very broad; the other corresponds to the situation $(\Delta t=\mathrm{n} T \mathrm{c})$ when the atoms are at rest (the velocity distribution is narrow), but the displacement distribution is wide. In such way, we squeezed the uncertainties of either atomic displacement or momentum, depending on the phase delay between two TPBS excited by each pump [8].

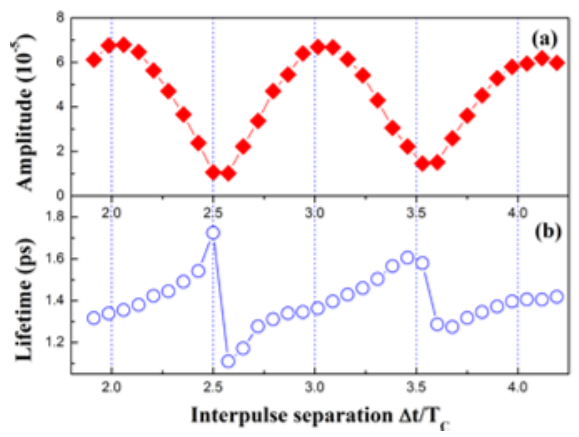

Fig. 2. Modulation of amplitude (a) and lifetime (b) of TPBS oscillations as a function of interpulse separation in the unit of the oscillation period $T \mathrm{c}=272 \mathrm{fs}$. The amplitude and lifetime are obtained from fitting the time-domain signals and extrapolating the oscillations to the zero delay time.

In contrast to the amplitude modulation which behaves classically, the observed modulation of lifetime is difficult to explain within a classical interference scheme. The change of lifetime observed in our experiments can be either correlated or anti-correlated to that of amplitude, and occurs over a shorter time scale as compared to its increase, which takes place more gradually. This modulation of lifetime, unequivocally suggests that we are dealing with quantum interference. Indeed, for classical interference, the change of coherent lifetime is impossible: classical coherent states are always transformed into different coherent states. The TPBS amplitude scales with the real part of squeeze parameter, whereas the oscillation lifetime measures how long the constituents of TPBS are correlated: the longer the time, the stronger the correlation.

\section{Conclusions}

Coherent two-phonon bound states have been excited impulsively in $\mathrm{ZnTe}(110)$ and controlled coherently by using two collinear pump pulses. Depending on the interpulse separation between pump beams, the quantum noise can be either enhanced or suppressed.

\section{References}

1. X. Hu, F. Nori, Phys. Rev. Lett. 76, 2294 (1996)

2. X. Hu, F. Nori, Physica B 263-264, 16 (1999)

3. G. A. Garrett, A. G. Rojo, A. K. Sood, J. F. Whitaker, R. Merlin, Science 275, 1638 (1997)

4. A. Bartels, T. Dekorsy, H. Kurz, Phys. Rev. Lett. 84, 2981 (2000)

5. Y. S. Lim, S. C. Yoon, K. J. Yee, J. H. Lee, D. S. Kim, D. Lee, Phys. Rev. B 68, 153308 (2003)

6. N. Vagelatos, D. Wehe, J. S. King, J. Chem. Phys. 60, 3613 (1974)

7. J. Hu, O. V. Misochko, K. G. Nakamura, Phys. Rev. B 84, 224304 (2011)

8. O. V. Misochko, J. Hu, K. G. Nakamura, Phys. Lett. A 375, 4141 (2011) 